\title{
Life-threatening bleeding of a duodenal gastrointes- tinal stromal tumor in a teenager: a rare case report
}

Authors

Institutions
Piero V. Valli ${ }^{1}$, Carlo Valli ${ }^{2}$, Thomas Pfammatter ${ }^{3}$, Peter Bauerfeind

${ }^{1}$ Clinic of Gastroenterology and Hepatology, University Hospital Zürich, Switzerland

${ }^{2}$ Department of Internal Medicine, Gastroenterology, Spitalzentrum Oberwallis, Visp, Switzerland

${ }^{3}$ Institute of Diagnostic and Interventional Radiology, University Hospital Zürich, Switzerland submitted 20. May 2016 accepted after revision 29. July 2016

\section{Bibliography}

Dol http://dx.doi.org/

10.1055/s-0042-115936

Published online: 10.11.2016

Endoscopy International Open 2016; 04: E1244-E1246

(c) Georg Thieme Verlag KG

Stuttgart · New York

E-ISSN 2196-9736

\section{Corresponding author}

Prof. Dr. med. Peter Bauerfeind

University Hospital of Zurich

Clinic of Gastroenterology and Hepatology

Raemistrasse 100

Zürich ZH 8091

Switzerland

Phone: +41-44-255-3642

Fax: +41-44-255-9933

peter.bauerfeind@usz.ch
Duodenal gastrointestinal stromal tumors (GIST) are per se infrequent and are exceptional in children or young adults. So far, only 2 cases of pediatric duodenal GISTs have been published. Here we report on the case of a 19-year-old female patient who was admitted in hemorrhagic shock due to arterial bleeding of a duodenal GIST located in immediate proximity to the major duodenal papilla. After several attempts of endoscopic hemostasis failed, the tumor bleeding was controlled with a second coil embolization of the pancreaticoduodenal arcades.

\section{Introduction}

\section{$\nabla$}

Gastrointestinal stromal tumors (GIST) are rare mesenchymal and typically subepithelial neoplasms that account for approximately $1 \%$ of the gastrointestinal tumors [1]. Predilection sites are the stomach and the small intestine [2]. Duodenal GISTs are even more rare and exceptional in children or young adults. GISTs most commonly originate from the interstitial cells of Cajal (ICC) in the fourth layer of the gastrointestinal tract wall (muscularis propria). Diagnosis is confirmed by expression of positive immunohistochemical staining for CD117 (KIT receptor tyrosine kinase, c-KIT protein), which is found in $95 \%$ of cases. CD34 stains positive in $70 \%$ of GIST. The overall GIST incidence is estimated to range between 10 to 20 cases per million among the adult population [1]. GISTs in childhood either occur sporadically or in the context of hereditary syndromes like neurofibromatosis type 1 (NF1) or CarneyStratakis syndrome. Nevertheless, the occurrence of sporadic duodenal GISTs in children and young adults is exceedingly low. A literature search revealed that only 2 cases of duodenal GISTs in children have been reported $[3,4]$. Here we report on the case of a 19-year old female patient who was admitted in hemorrhagic shock due to suspected gastrointestinal bleeding.

\section{Case report \\ $\nabla$}

A 19-year-old otherwise healthy female tourist was admitted to a secondary care hospital after fainting while skiing due to suspected gross blood loss with an initial hemoglobin level of $60 \mathrm{~g} / \mathrm{L}$. The patient developed tarry stools during the hospitalization. After volume resuscitation including red blood cell (RBC) transfusions, a tumorous mass with a central bleeding ulceration (bull's eye appearance, Fig. 1) was diagnosed upon emergency endoscopy. The submucosal tumor bulging into the duodenal lumen was found in immediate proximity to the major duodenal papilla ( Fig. 2). Blood oozing was noted and primary hemostasis was accomplished by injection of saline-diluted epinephrine and the application of 2 Instinct $^{\mathrm{TM}}$ endoscopic hemoclips. Non-contrast-enhanced computed tomography failed to locate the primary tumor and did not reveal any distant metastasis. After overnight observation, the patient was transferred to our tertiary care hospital for further diagnostic work-up.

Upon arrival at our institution, endosonography demonstrated a submucosal, hypervascular and hypoechoic tumor. The neoplasm with a central bleeding vessel arose from the muscularis propria (fourth wall layer) and measured $25 \times 15 \mathrm{~mm}$ ( $\bullet$ Fig. 3 and $\bullet$ Fig.4). Our initial differential diagnosis based on clinical presentation and endosonographic imaging consisted of gastric stroma tumor (GIST), neuroendocrine tumor (NET), gangliocytic paraganglioma [5], leiomyoma [6] and solid pseudo-papillary tumor of the pancreas [7]. In addition to endosonography-guided fine-needle aspiration, regular biopsies were harvested and an on-site cytologist ensured attainment of diagnostic tissue.

Recurrent tumor bleeding after tissue harvesting was then stopped by application of Hemospray ${ }^{\circledR}$. After observing a recurrent decrease in hemoglobin levels during the following night, ongoing tu- 


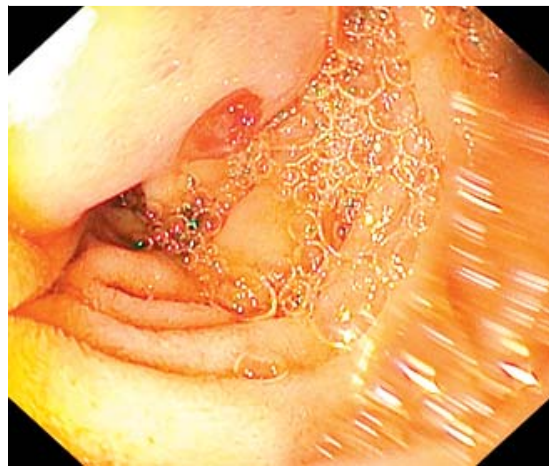

Fig. 1 Duodenal tumorous mass with central vessel bulging into the lumen (bull's eye appearance).

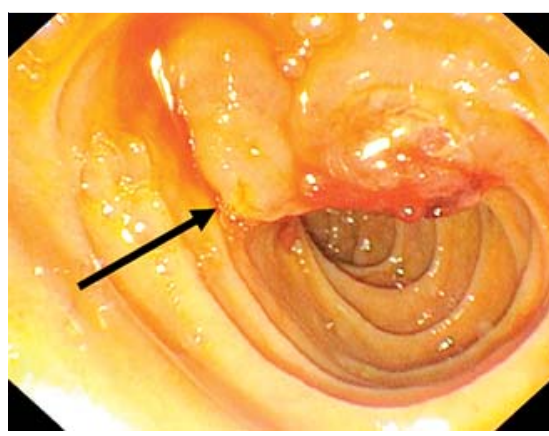

Fig. 2 Bleeding duodenal mass adjacent to the major duodenal papilla (black arrow).

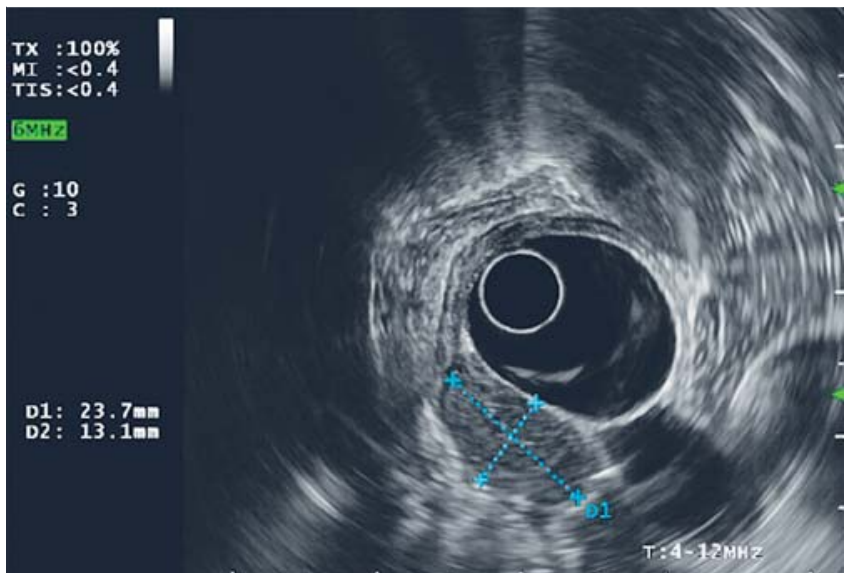

Fig. 3 Submucosal, hypoechoic tumor of the duodenum.

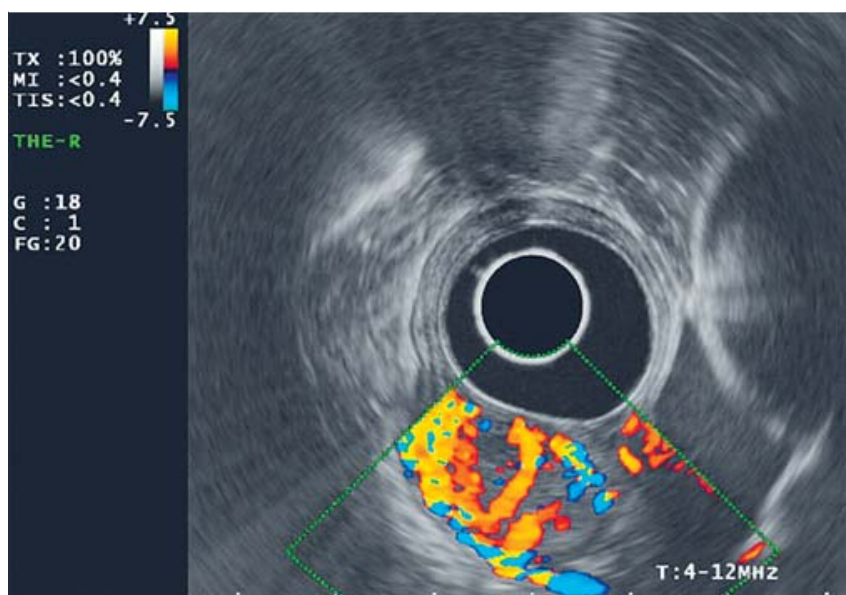

Fig. 4 Hypervascular submucosal tumor of the duodenum.

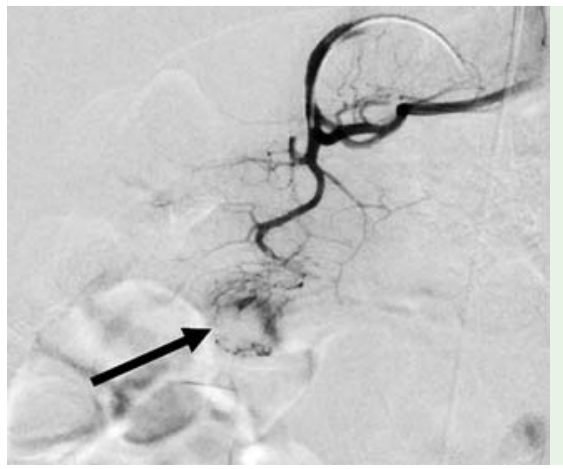

Fig. 5 Hypervascula tumor (black arrow) of the duodenum predominantly supplied by the anterior pancreatoduodenal arcade.

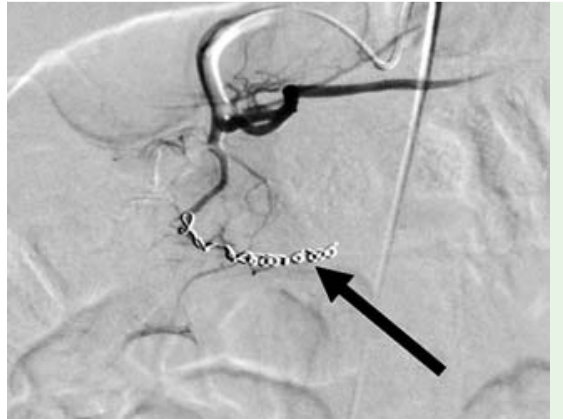

Fig. 6 Coil embolization of the superior pancreaticoduodenal arteries. mor bleeding was confirmed by upper gastrointestinal endoscopy. Given the lack of further endoscopic hemostasis options, transarterial coil embolization of the tumor-supplying anterior pancreaticoduodenal arcade was performed ( $\bullet$ Fig. 5 and $\bullet$ Fig. 6 ). Despite the first coil embolization, persistent blood loss was noted overnight in the patient. Intermittent bleeding was confirmed by duodenoscopy and no permanent hemostasis was achieved by Gold Probe ${ }^{\mathrm{TM}}$ coagulation. Repeat angiography showed persistent tumor staining through tiny branches of the posterior pancreaticoduodenal arcade. The bleeding was finally halted by coil embolization of the inferior pancreaticoduodenal artery via the superior mesenteric artery and the origin of the posterior arcade via the gastroduodenal artery. The diagnosis of a GIST was ultimately established by positive staining for CD117 (cKit), CD34 and DOG-1 and negative staining for SMA und S100 PanCK B.

After no further bleeding was detected over the course of the next 48 hours, the patient was transferred to a center in her home country for further treatment. After an initial trial of tumor downsizing with imatinib, the medication had to be discontinued due to severe adverse events including vomiting, diarrhea, fatigue and severe bone marrow suppression requiring multiple transfusions. Thereafter, the GIST was removed entirely by a pancreas-sparing duodenectomy. The final histology report of the in toto resected GIST (positive staining for CD117, CD34 and DOG1; negativity for Desmin, S11 and CD31) displayed 3 mitoses/50 HPF. These findings correspond to a low risk (8.3\%) for progressive disease [8]. The further postoperative recovery of the patient has been uneventful so far.

\section{Discussion}

We here report the third case in literature of a duodenal GIST in a patient younger than age 20. The initial presentation of the tumor in our teenage patient was a life-threatening arterial tumor bleeding with consecutive hemorrhagic shock. Even though pediatric GISTs frequently present with gastrointestinal bleeding 
[9], the highly delicate duodenal location in immediate proximity to the major duodenal papilla rendered the hemostasis very challenging in our case. In contrast to our case, the vast majority of previously reported bleeding pediatric GISTs were located in the stomach and therefore potentially more easily accessible for endoscopic or surgical hemostasis $[10,11]$.

Given the challenging tumor location adjacent to the Papilla of Vater, we sought to establish a histopathologic diagnosis before performing an extensive surgical procedure such as pancreaticoduodenectomy (Whipple's procedure) on our patient. While awaiting definite histopathologic diagnosis including immunohistological staining, all conventional endoscopic hemostasis procedures (including epinephrine injection, classical and Instinct $^{\mathrm{TM}}$ endoscopic hemoclip application, argon plasma coagulation, Gold Probe ${ }^{\mathrm{TM}}$ coagulation and application of Hemospray ${ }^{\circledR}$ ) failed to adequately control the life-threatening tumor bleeding. Fearing a complete closure of the common biliary and the pancreatic duct, OTSC ${ }^{\circledR}$ application on the juxtapapillary lesion was rejected. Ultimately, only a second transarterial coil embolization stopped the intermittent arterial GIST bleeding permanently and allowed stabilization of the patient for repatriation to her home country for definite resection (bridge to resection).

Although some authors claim that pediatric GISTs are a different entity with variable local and systemic tumor behavior than adult GISTs [9], complete endoscopic or surgical resections (R0) also remain the cornerstone for pediatric GIST treatment. Depending on tumor size and mitotic rate, risk of recurrence and metastasis rate can be estimated and an individual treatment plan needs to be created, including optional adjuvant treatment with the tyrosine receptor inhibitor imatinib and appropriate surveillance frequency [1].

Despite the very rare GIST occurrence in children and young adults, it should be included in the differential diagnosis for gastrointestinal blood loss, iron deficiency anemia and unexplain- able gastrointestinal pain. Given the fairly high incidence of metastasis (approx. 50\%) and the elevated recurrence rate, early diagnosis of GIST is even more crucial in young patients with a potentially long life expectancy [12].

\section{Competing interests: None}

\section{References}

1 Miettinen M, Lasota J. Gastrointestinal stromal tumors-definition, clinical, histological, immunohistochemical, and molecular genetic features and differential diagnosis. Virchows Arch 2001; 438: 1-12

2 Fletcher $C D$, Berman JJ, Corless $C$ et al. Diagnosis of gastrointestinal stromal tumors: A consensus approach. Hum Pathol 2002; 33: 459-465

3 Chiarugi M, Galatioto C, Lippolis P et al. Gastrointestinal stromal tumour of the duodenum in childhood: a rare case report. BMC Cancer 2007; $7: 79$

4 Hughes JA, CookJV, Said A et al. Gastrointestinal stromal tumour of the duodenum in a 7-year-old boy. Pediatr Radiol 2004; 34: 1024-1027

5 Boeriu A, Dobru D, Georgescu $R$ et al. Gangliocytic paraganglioma: a rare cause of gastrointestinal bleeding. J Gastrointestin Liver Dis 2015; 24: 109-112

6 Shetty SM, Kalokhe S, Rathi P et al. Duodenal leiomyoma - a rare cause of haematemesis. J Assoc Physicians India 2001; 49: 1114-1115

7 Apostolidis S, Papavramidis TS, Zatagias A et al. Hematemesis, a very rare presentation of solid pseudo-papillary tumors of the pancreas: a case report. J Med Case Rep 2008; 2: 271

8 Miettinen M, Lasota J. Gastrointestinal stromal tumors: pathology and prognosis at different sites. Semin Diagn Pathol 2006; 23: 70-83

9 Kaemmer DA, Otto J, Lassay L et al. The gist of literature on pediatric GIST: review of clinical presentation. J Pediatr Hematol Oncol 2009; 31: $108-112$

10 Dave M, Jimenez A, Evans $K$ et al. Treatment of recurrent pediatric gastrointestinal stromal tumors. Gastrointest Cancer Res 2012; 5: 139 140

11 Miranda ME, Alberti LR, Tatsuo ES et al. Gastrointestinal stromal tumor of the stomach in a child with a 3-year follow-up period - Case report. Int J Surg Case Rep 2011; 2: 114-117

12 Gold JS, van der Zwan SM, Gonen M et al. Outcome of metastatic GIST in the era before tyrosine kinase inhibitors. Ann Surg Oncol 2007; 14: $134-142$ 